\title{
Interlayer Forces and Ultralow Sliding Friction in Multiwalled Carbon Nanotubes
}

\author{
A. Kis, ${ }^{1,2,3}$ K. Jensen, ${ }^{1,3}$ S. Aloni, ${ }^{1,2,4}$ W. Mickelson, ${ }^{1}$ and A. Zettl ${ }^{1,2,3,4, *}$ \\ ${ }^{1}$ Department of Physics, University of California at Berkeley, Berkeley, California 94720, USA \\ ${ }^{2}$ Materials Sciences Division, Lawrence Berkeley National Laboratory, Berkeley, California 94720, USA \\ ${ }^{3}$ Center of Integrated Nanomechanical Systems (COINS), University of California at Berkeley, Berkeley, California 94720, USA \\ ${ }^{4}$ Molecular Foundry, Lawrence Berkeley National Laboratory, Berkeley, California 94720, USA
}

(Received 30 March 2006; published 11 July 2006)

\begin{abstract}
We describe interlayer force measurements during prolonged, cyclic telescoping motion of a multiwalled carbon nanotube. The force acting between the core and the outer casing is modulated by the presence of stable defects and generally exhibits ultralow friction, below the measurement limit of $1.4 \times$ $10^{-15} \mathrm{~N} /$ atom and total dissipation per cycle lower than $0.4 \mathrm{meV} /$ atom. Defects intentionally introduced in the form of dangling bonds lead to temporary mechanical dissipation, but the innate ability of nanotubes to self heal rapidly optimizes the atomic structure and restores smooth motion.
\end{abstract}

DOI: 10.1103/PhysRevLett.97.025501

Understanding atomic scale mechanical interactions, possibly leading to friction and dissipation remains a major challenge for a broad base of scientists and is an essential requirement for the development of viable nanoelectromechanical systems (NEMS). In this context, multiwalled carbon nanotubes (MWNTs) [1] have been proposed as an intriguing system for studying friction on the nanoscale [2-4] because of their constrained geometry, inert ultrasmooth surfaces and the small intershell distance that prevents the accumulation of contaminant particles. These features could make MWNTs the ideal building blocks for the fabrication of nanoelectromechanical devices like nanoscale motors [5], springs [3], variable resistors [6], or switches [7].

Theoretical modeling predicts that geometrically perfect MWNTs would be the "smoothest bearings" [2,4], with vanishingly small interlayer friction due to the suppression of collective stick-slip motion in incommensurate tube pairs, similarly to superlubricity in graphite [8]. However, as-produced carbon nanotubes do not generally possess the ideal, defect-free structure postulated in theoretical models. Various topological defects like vacancies [9], StoneWales defects [10], and adatoms [11] inevitably introduced during MWNT growth might lead to dissipation during intershell sliding in MWNTs. Even in topologically perfect nanotubes, thermally induced fluctuations [12] or phonon excitation due to interaction of the core with the edge of the outer casing [13] could lead to mechanical dissipation that would scale with the length of the moving section. Other theoretical models predict nonextensive interlayer friction, with dissipation coming from the movement of the core's fractured end [14] or phonon excitation due to interaction of the core with the edge of the outer casing [15].

A limited number of previous experiments on nanotube intershell sliding have yielded encouraging, though quantitatively incomplete results, based on estimates [3] or single-shot extraction experiments in scanning electron microscopes [16,17], whereas cyclic motion is required in order to distinguish between dissipative and conserva-
PACS numbers: $62.25 .+\mathrm{g}$, 61.46.Fg, 81.40.Pq

tive interlayer forces. This leaves it unclear if the predicted ultra-low-friction properties of nanotubes can be realized in practice.

In order to quantify the dynamic properties of interlayer sliding in MWNTs, we have performed measurements inside a JEOL-2010 transmission electron microscope (TEM) using a nanomanipulation stage with a force sensor, Fig. 1(a). A whisker of arc discharge grown tubes was glued onto a Pt wire and mounted in the mobile, sample part of the manipulator. Outer layers are removed by electrical breakdown [18], exposing a core which is spot welded to the force sensor, a Pt-coated atomic force microscope (AFM) tip, using a $5 \mathrm{~V}$ pulse, current limited to below $10 \mu \mathrm{A}$ [3]. The outer casing is in stable mechanical contact with a Pt wire mounted on the nanomanipulation stage. The piezodriven actuator induces cyclic telescopic motion of the core relative to the casing [Fig. 1(b)] with frequencies in the $0.1-100 \mathrm{~Hz}$ range. Relative positions of the core, casing, and AFM cantilever with a spring constant of $0.26 \mathrm{~N} / \mathrm{m}$ are directly observed in the TEM and digitized from video recording using pattern recognition software. Depending on the TEM magnification, displacement and force resolutions of $1.6 \AA$ and $40 \mathrm{pN}$ can be achieved in this way.

Assuming for the sake of generality that the interlayer friction is proportional to the contact area and characterized by shear strength $\tau$ [19], the expected force acting between neighboring MWNT layers can be written as the sum of the interlayer friction and cohesion force:

$$
\begin{aligned}
F & =F_{\text {friction }}+F_{\text {cohesion }} \\
& =\underbrace{-\operatorname{sgn}(\dot{x}) \pi D \tau L(x)}_{\text {dissipative }} \underbrace{-\gamma \pi D+f_{\text {corr }}(x)}_{\text {conservative }},
\end{aligned}
$$

where $f_{\text {corr }}$ is the expected oscillating contribution to cohesion due to interlayer corrugation $[4,20], L$ is the interlayer overlap length dependent on the telescoping core position $x, D$ the diameter of the mobile core, and $\gamma$ the surface energy of the MWNT shell. The average value of 


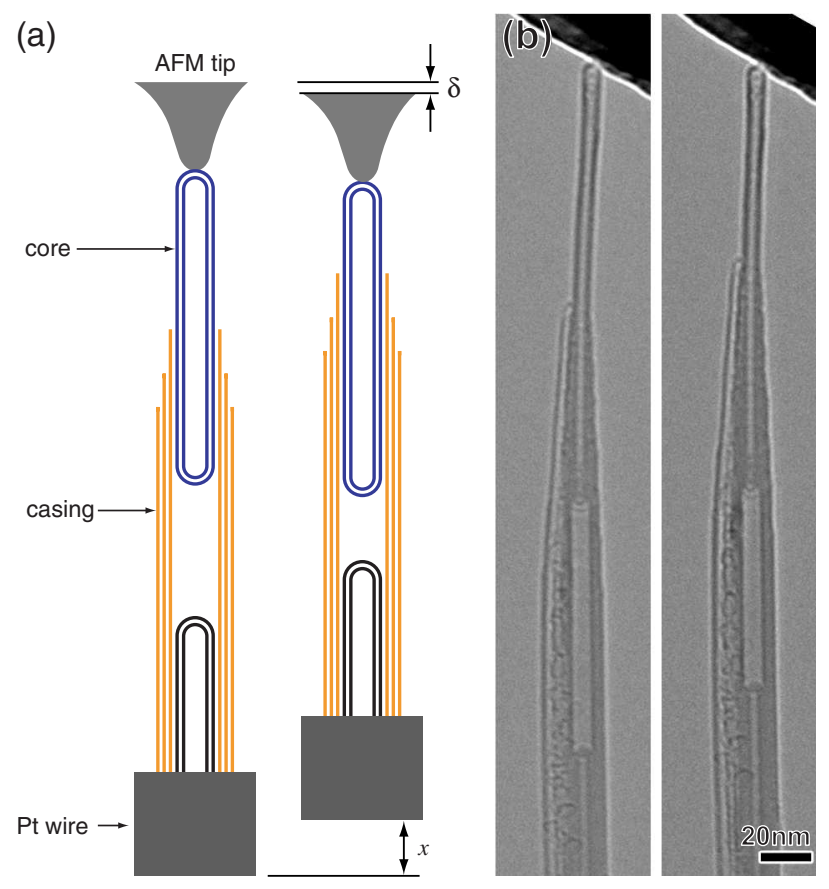

FIG. 1 (color online). (a) Schematic drawing of the measurement setup. The protruding core of a MWNT is connected to an AFM tip. The outer casing is mechanically connected on the other end to a Pt wire, attached onto a piezoscanner which is used to induce cyclic telescoping motion. The force acting between the casing and the core is monitored by measuring the deflection $\delta$ of the AFM cantilever which is much smaller than the casing displacement $x$. (b) Two TEM images showing a telescoping MWNT.

the cohesion force $\gamma \pi D$ can be experimentally determined at the end of the experimental run by complete extraction of the core from the casing. Friction and dissipation would be manifested by a nonvanishing closed loop integral of $F d x$, where $F$ is the total interlayer force, Eq. (1), leading to hysteresis in force-displacement curves with different force averages during inward and outward motion of the core, requiring net work to be performed on the system during one cycle of motion.

The measured force acting between the core and the casing during cyclic telescoping motion, Fig. 2(a), shows an irregular force profile with an average value that corresponds to the interlayer cohesion which acts as a constant force spring providing the restoring force acting on the core [3]. The fluctuations in force are on the order of $100 \mathrm{pN}$, devoid of periodicity and with peaks of subnanometer widths, Fig. 2(a). A color-coded force map corresponding to 14 consecutive cycles of motion, shown on Fig. 2(b) with the first cycle at the bottom, clearly demonstrates that the force profile is reproducible and independent of the direction of motion. Surprisingly, the force is far more irregular than the theoretically predicted behavior of perfect nanotubes $[4,20]$ expected to result in regular force oscillations with periodicity that corresponds with the periodicity of the registry-dependent interlayer poten- tial energy. The reproducible nature of observed force fluctuations indicates that they might be due to randomly distributed, stable defects that locally modify the interface geometry between the core and casing thus modulating the interlayer force. The obvious candidate for such defects is the Stone-Wales (SW) defect [10], consisting of two heptagons and two pentagons created by a $90^{\circ} \mathrm{C}-\mathrm{C}$ bond rotation. This is the most common and stable form of defects in carbon nanotubes. Chemisorbed impurities are less likely due to constrained space between the neighboring shells. The amplitude and spatial extent of measured force fluctuations are consistent with theoretical calculations for either SW defects on the core interacting with the open end of the casing or SW defects on the casing interacting with the capped core [21]. The fluctuating force could therefore represent a unique "fingerprint" of the telescoping nanotube, reflecting the spatial arrangement of stable defects, probed by mechanical means.

Most importantly, an irregular force profile does not necessarily imply finite friction and dissipation. Even though the force acting on the core during one cycle of motion is irregular, it is, for the majority of traces, independent of the direction of motion, Fig. 2, with equal force averages in both directions. For the majority of traces we observe, the cyclical telescoping motion of nested nanotubes is nonhysteretic and therefore frictionless and dissipation-free, within the measurement limit. Based on the precision of our instrumentation, we estimate that the friction is below $1.4 \times 10^{-15} \mathrm{~N} /$ atom, corresponding to a shear strength $\tau$ lower than $0.05 \mathrm{MPa}$ and total dissipated energy per cycle of less than $0.4 \mathrm{meV} /$ atom. This is more than an order of magnitude lower than the previous estimate of $2.3 \times 10^{-14} \mathrm{~N} /$ atom $(0.4 \mathrm{MPa})$ on the interlayer friction (shear strength) [3] and lower than the observed friction for one of the least dissipative nanoscale interfaces - the incommensurate contact between $\mathrm{C}_{60}$ islands and $\mathrm{NaCl}$ [22]. We thus find that, despite the presence of stable defects leading to an irregular force profile, telescoping nanotubes need not display mechanical dissipation, demonstrating that "real-world" nanotubes could be used to construct defect-tolerant linear nanomachines with high performance.

How robust is the ultrasmooth motion of telescoping nanotubes? Prominent horizontal dark and bright streaks close to the top of the force map shown on Fig. 2(b) indicate temporary occurrences of hysteresis, implying that interlayer sliding is not completely immune to friction. Such hysteretic behavior, shown in more detail on Fig. 2(c), can occur spontaneously without any intervention on our part and is reminiscent of atomic friction $[23,24]$. As it turns out, the hysteretic behavior is very short lived as the nanotube is cycled. As an example, for the upper traces in Figs. 2(b) and 2(c), the original force signature with no observable friction is restored after only two cycles of dissipative motion.

The short period of dissipative motion described above can be attributed to the formation of at least one vacancy on 

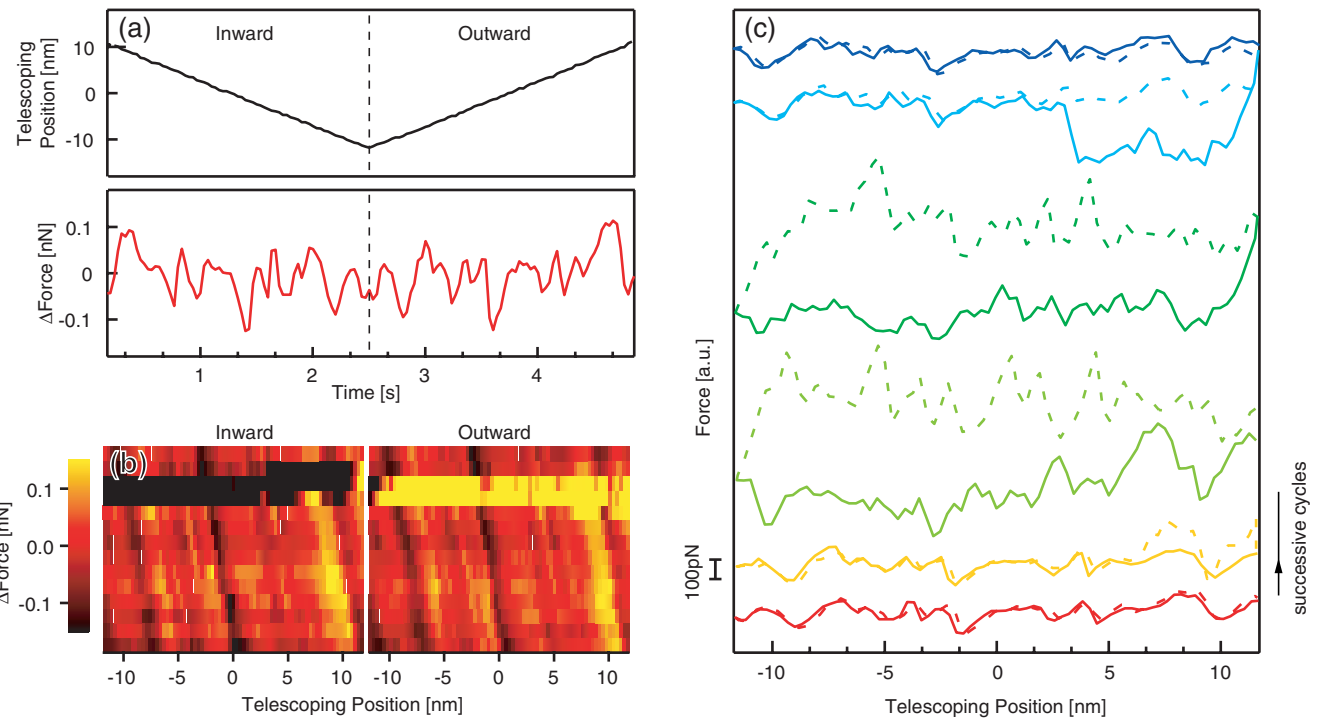

FIG. 2 (color online). (a) Telescoping core position and force acting on it during cyclic actuation for both directions of movement. The force profile is irregular with fluctuations on the order of $100 \mathrm{pN}$. (b) Color-coded force map for 14 cycles of motion shows the reproducibility of the irregular force profile. Dark and bright horizontal streaks indicate the short-lived occurrence of mechanical dissipation. (c) A detailed plot of the last 6 sequential cycles of telescoping motion from part (b) showing spontaneous appearance and disappearance of friction. Full and dashed lines represent the inward and outward motion of the core. Curves are offset for clarity.

the telescoping interface, leading to the appearance of friction and increased interlayer force that is consistent with previous theoretical modeling [9]. However, rapid healing through dangling bond saturation and StoneWales defect formation [25], promoted by telescoping movement, optimizes the atomic structure and restores the original force signature, Fig. 2(c). This demonstrates that carbon nanotubes are endowed with the fascinating ability to self-repair, absorbing damage that could otherwise lead to dissipation and jamming. Apart from such short, transient excursions, dissipation-free telescoping motion of this nanotube continued for up to $30 \mathrm{~min}$, without any permanent changes.

Under our imaging conditions, the $100 \mathrm{keV}$ electron beam used for TEM imaging with an approximate flux of
$0.4 \mathrm{~A} / \mathrm{cm}^{2}$ can cause limited knock-on damage, displacing carbon atoms from the nanotube lattice, thus creating vacancies with associated dangling bonds and interstitials. Assuming a displacement energy of $17 \mathrm{eV}$ [26], our imaging conditions will result in at least $5 \times 10^{-5}$ displacements per atom every second (dpa/s), with $3 \mathrm{dpa} / \mathrm{s}$ for a typical telescoping interface containing $6 \times 10^{4}$ atoms. Cycles with dissipation are rarely observed, implying that continuous telescoping motion itself can suppress creation of covalent bonds in the interface between the core and the casing. Stopping the telescoping motion for only a couple of seconds can lead to permanent jamming of the core due to $e$-beam radiation induced crosslinking [27] with the case.

After prolonged exposure to the TEM electron beam, the telescoping MWNT system becomes permanently dam-
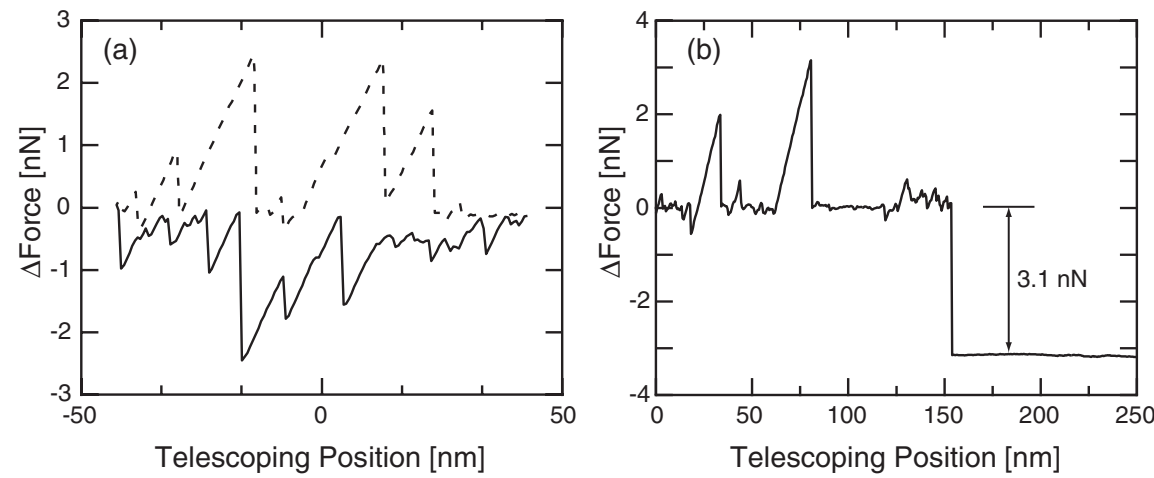

FIG. 3. (a) An example of high amplitude telescoping motion exhibiting mechanical dissipation events with no apparent periodicity. Full and dashed lines represent inward and outward motion of the core with respect to the outer casing. (b) Dependence of force on the telescoping position during slow pullout. Sharp, stick-slip-like features are superposed on the background due to the interlayer adhesion. Complete extraction of the core is manifested by a sudden drop of force. 
aged as the self-healing mechanism is no longer able to cope with the high disorder concentration and restore dissipation-free movement. An example of a finite friction loop from such a damaged tube is shown on Fig. 3(a). The telescoping nanotube geometry with its variable surface area provides an unprecedented opportunity to confirm that the friction, when it occurs, is due to localized defects giving rise to dissipation events and not to area dependent atomic friction [19]. In addition, complete extraction of the core from the casing allows us to determine the intershell cohesive force for damaged tubes. During slow pullout $(15 \mathrm{~nm} / \mathrm{s})$, the force is found to be characterized by a constant background with superimposed abrupt dissipation events, shown in Fig. 3(b). Magnitudes of these events range from $0.3-3.1 \mathrm{nN}$, similar to those in the friction loop shown on Fig. 3(a). Complete extraction of the core from the outer casing manifests itself as an abrupt decrease of force, with a magnitude of $3.1 \mathrm{nN}$, Fig. 3(b). This corresponds to a surface energy of $\gamma=0.2 \mathrm{~J} / \mathrm{m}^{2}$ ( $33 \mathrm{meV} /$ atom), within the range of values we found for undamaged tubes $\left(0.14-0.2 \mathrm{~J} / \mathrm{m}^{2}\right)$ and in good agreement with theoretical predictions $[2,3,28]$. According to Eq. (1), the presence of static shear force, dependent on overlap length, would give rise to a length-dependent total force. The pullout force shows no observable length variation, indicating that the static shear strength is lower than $0.04 \mathrm{MPa}$, even in tubes severely damaged by irradiation. Mechanical dissipation is therefore mainly due to the presence of externally induced defects leading to instabilities in motion. In the absence of mechanical instabilities, atomic friction, dependent on the contact area, is dramatically suppressed. Such suppression of friction was first reported for $\mathrm{NaCl}$ surfaces in ultrahigh vacuum [29].

Our measurements of interlayer forces provide new insights into the fundamental properties of interlayer interaction in MWNTs. Van der Waals interaction between neighboring layers of MWNTs is strongly modulated by defects that are inevitably introduced during nanotube growth, yet the motion exhibits ultralow friction. Sliding nanotubes also have the surprising ability to absorb induced damage by a self-healing mechanism that preserves the absence of detectable mechanical dissipation.

We would like to thank Steve Butler and Andrew Mei from U.C. Berkeley Physics department and the LBNL machine shops for work on the TEM holder. Paul Tangney is gratefully acknowledged for illuminating discussions and Christian Esquivias for nanotube synthesis. A. K. thanks the Swiss National Science Foundation (SNF) for their financial support. This work was partially supported by the National Science Foundation and the Office of Energy Research of the U.S. Department of Energy.

*Electronic address: azettl@berkeley.edu

[1] S. Iijima, Nature (London) 354, 56 (1991).
[2] J.-C. Charlier and J.-P. Michenaud, Phys. Rev. Lett. 70, 1858 (1993).

[3] J. Cumings and A. Zettl, Science 289, 602 (2000).

[4] A. N. Kolmogorov and V. H. Crespi, Phys. Rev. Lett. 85, 4727 (2000).

[5] A.M. Fennimore, T.D. Yuzvinsky, W. Q. Han, M.S. Fuhrer, J. Cumings, and A. Zettl, Nature (London) 424, 408 (2003).

[6] J. Cumings and A. Zettl, Phys. Rev. Lett. 93, 086801 (2004).

[7] L. Forro, Science 289, 560 (2000).

[8] M. Dienwiebel, G. S. Verhoeven, N. Pradeep, J. W. M. Frenken, J. A. Heimberg, and H.W. Zandbergen, Phys. Rev. Lett. 92, 126101 (2004).

[9] M. Huhtala, A. V. Krasheninnikov, J. Aittoniemi, S. J. Stuart, K. Nordlund, and K. Kaski, Phys. Rev. B 70, 045404 (2004).

[10] A. J. Stone and D. J. Wales, Chem. Phys. Lett. 128, 501 (1986).

[11] A. A. El-Barbary, R. H. Telling, C.P. Ewels, M.I. Heggie, and P. R. Briddon, Phys. Rev. B 68, 144107 (2003).

[12] J. Servantie and P. Gaspard, Phys. Rev. Lett. 91, 185503 (2003).

[13] Y. Zhao, C.-C. Ma, G. Chen, and Q. Jiang, Phys. Rev. Lett. 91, 175504 (2003).

[14] Z. Xia and W. A. Curtin, Phys. Rev. B 69, 233408 (2004).

[15] P. Tangney, S. G. Louie, and M. L. Cohen, Phys. Rev. Lett. 93, 065503 (2004).

[16] S. Akita and Y. Nakayama, Jpn. J. Appl. Phys. 42, 3933 (2003).

[17] M. F. Yu, B. I. Yakobson, and R. S. Ruoff, J. Phys. Chem. B 104, 8764 (2000).

[18] J. Cumings, P. Collins, and A. Zettl, Nature (London) 406, 586 (2000).

[19] F. Bowden and D. Tabor, The Friction and Lubrication of Solids (Oxford University, Oxford, 1950).

[20] W. Guo, Y. Guo, H. Gao, Q. Zheng, and W. Zhong, Phys. Rev. Lett. 91, 125501 (2003).

[21] W. Guo, W. Zhong, Y. Dai, and S. Li, Phys. Rev. B 72, 075409 (2005).

[22] R. Luthi, E. Meyer, H. Haefke, L. Howald, W. Gutmannsbauer, and H.J. Guntherodt, Science 266, 1979 (1994).

[23] E. Gnecco, R. Bennewitz, T. Gyalog, and E. Meyer, J. Phys. Condens. Matter 13, R619 (2001).

[24] G. A. Tomlinson, Philos. Mag. 7, 905 (1929).

[25] P. M. Ajayan, V. Ravikumar, and J.-C. Charlier, Phys. Rev. Lett. 81, 1437 (1998).

[26] V.H. Crespi, N. G. Chopra, M. L. Cohen, A. Zettl, and S. G. Louie, Phys. Rev. B 54, 5927 (1996).

[27] A. Kis, G. Csanyi, J.-P. Salvetat, T.-N. Lee, E. Couteau, A. J. Kulik, W. Benoit, J. Brugger, and L. Forro, Nat. Mater. 3, 153 (2004).

[28] L. X. Benedict, N. G. Chopra, M. L. Cohen, A. Zettl, S. G. Louie, and V.H. Crespi, Chem. Phys. Lett. 286, 490 (1998).

[29] A. Socoliuc, R. Bennewitz, E. Gnecco, and E. Meyer, Phys. Rev. Lett. 92, 134301 (2004). 